\title{
CLUSTER BASED ARCHITECTURE FOR LOCALIZATION AND TRACKING IN WIRELESS SENSOR NETWORK
}

\author{
${ }^{1}$ Prabhakaran, P. and ${ }^{2}$ Nelson Kennedy Babu \\ ${ }^{1}$ Department of CSE, Vivekanandha College of Engineering for Women, TamilNadu, India \\ ${ }^{2}$ CMS College of Engineering, TamilNadu, India
}

Received 2013-12-18; Revised 2013-12-27; Accepted 2014-01-17

\begin{abstract}
Wireless sensor networks are an efficient way of reading and transmitting information through a system of controls. They are used for a variety of purposes. The signals passed between each sensing unit and the central control unit needs to be theoretically nonexistent for perfect transmission but there are many hindrances like transmission techniques and physical disturbances which cause delay. Here we discuss cluster based and prediction based tracking mechanisms to control and make the process more efficient.
\end{abstract}

Keywords: Signal, WSN, Tracking, Transmission Techniques

\section{INTRODUCTION}

\subsection{Wireless Sensor Network}

A Wireless Sensor Network (WSN) is defined as a combination of huge sum of sensor nodes. In WSN Sensor nodes are densely deployed. Generally these sensor nodes are seen as sensing devices that can be used to display conditions as temperature, sound, vibration, pressure, motion or pollutants at various locations. Generally sensor nodes are small, inexpensive, these devices are limited in power computation capacities and memory and they may not have global identification (Watfa and Commuri, 2006; Khin, 2008).

Wireless Sensor Networks (WSNs) are being planned and advanced for many types of claims involving monitoring and management of the physical world in a bind less fashion normally, each individual sensor node be able to sense in various modalities but has limited signal processing and communication capability (Wang et al., 2007). There are a lot of researches going on with WSNs (Manisekaran et al., 2012) which talks on energy consumption related stuff. But, what we aim at this research makes it special and unique in its kind.

\subsection{Target Tracking in WSN}

\subsubsection{What is Tracking?}

Target tracking is considered to be one of the most important roles of the wireless sensor network (Watfa and Commuri, 2006; Khin, 2008; Raza et al., 2009), which is delay responsive and needs the information to be transmitted to a central controller reliably within a definite deadline. In the applications of target tracking with WSNs, the users are normally concerned to know the position of target at each time interval.

Data reporting of the target is required. In continuous observation, sensor nodes in the network cooperate with each other in detecting the target, calculating the signal the target emitting and conveying measurements to the base-station for additional processing (Li and Ren, 2011).

\subsection{Need and Application of Target Tracking}

Object detection and tracking is an essential ability in many sensor network applications, such as in military it is use for stalking foe vehicles, detecting illegal border crossings, in civilian it is use for tracking the movement of wild animals in wildlife conserve, environmental monitoring (e.g., traffic, habitat and security), health Corresponding Author: Prabhakaran, P., Department of CSE, Vivekanandha College of Engineering for Women, TamilNadu, India 
(Tua et al., 2009; Fuemmeler and Veeravalli, 2008). GPS is use for locating people and tracking the mobile objects globally (Cenedese et al., 2010). Since there are number of sensors in WSN so tracking is very important for locating node in network and getting data from that node.

\subsection{Types of Target Tracking}

There are basically two types of approaches of target tracking in WSNs, which are 1. Centralized approach and 2.distributed approach. In the Centralized approach the sensors in the network sense the target and send board signatures to the Base Station. Base Station determines whether there is a target is available or not by using the target signature which is sends by sensing node, if the target is found then it is tracked by Base Station. In the Distributed approach of Target Tracking the whole sensor network separated into areas, each area has a Manager Node. The processing tasks are performing at the manager node, not only at base station (Khin, 2008).

\subsection{Issues of Target Tracking}

Energy efficiency is also a main issue. Since nodes have limited battery power, so routing must be efficient to increase network lifetime. In addition limited energy, routing delay is another factor which affects the objective of transferring time critical information reliably across the network.

The ability of coverage area is main issue to track a dynamic target of the WSN (Watfa and Commuri, 2006).

The strength of physical signals decays with increase in distance, so readings of the sensor nodes have similar pattern if their distances to a target are approximately same. Therefore sensed data for targets is usually exhibits a large degree of redundancy $(\mathrm{Li}$ and Ren, 2011).

Network localization is an real idea to reduce the volume of conducted data, but not so much capable for multiple targets tracking which need high complexity computation (Li and Ren, 2011).

\section{LITERATURE REVIEW}

\subsection{Increasing Lifetime of Target Tracking Wireless Sensor Networks (Khin, 2008)}

Energy consumption by sensor node is addressed in this study. Since the sensor node have limited energy source so energy should be use efficiently. In this study energy efficient heterogeneous wireless sensor network for target tracking is presented. Which is use for improve the network life time. In this study Static cluster-based architecture is use for increasing lifetime of target tracking in wireless sensor network and enables good localization outcome with low energy ingesting for each sensor in the network. In this approach cluster use two operation mode Active mode and Sleep mode. This architecture devours less energy and increases lifetime than centralized and dynamic clustering architectures. The performance matrices uses in this study are energy consumption and lifetime. The drawbacks of this study are that is this study use acoustic sensor for tracking target, It is not discussed what happen when a target is at same distance of acoustic sensor. Time delay is also not discussed. Other performance matrices are not discussed.

\subsection{Compressing Information of Target Tracking in Wireless Sensor Networks $(\mathbf{L i}$ and Ren, 2011)}

In this study data implosion and redundancy due to traffic load is addressed. In this study data compressing based target tracking protocol is proposed for reducing traffic load in network. This approach first adds on a clustering based data gather method to group sensor nodes into clusters, after that a novel threshold technique with bounded error is proposed which exploit the spatial correlation of sensed data and compress the data in the same cluster. This study introduce new approximation scheme which makes full use of correlations among data of multiple sensor nodes. The performance metrics uses in this study are-overhead which is lowering and error in tracking result. This study is not suitable for tracking moving object. In this study when a target is sensed by sensor then sensor sense data to cluster head and cluster head fire a waiting time $\mathrm{T}_{\mathrm{w}}$ to collect packet from nodes which causes delay in network.

\subsection{Smart Sleeping Policies for Energy Efficient Tracking in Sensor Networks (Fuemmeler and Veeravalli, 2008)}

In this study author address tracking problem of an object when it is moving randomly through a dense network of wireless sensor. In this study researcher develop a framework for optimizing the tradeoff between energy cost and tracking error without assuming the use of wakeup radios. This is an example of a Partially Observable Markov Decision Process (POMDP). It provides simulation results that characterize the performance of our suboptimal policies. The performance matrices uses in this study are-tracking error is improved, energy is con-served. In this study the optimal solution of a chasing problem with drowsy node is not found. This study discuses tracking about a single target, multiple target tracking in this need to be discuss. 


\subsection{A Predictive Energy-Efficient Technique to Support Object-Tracking Sensor Networks (Samarah et al., 2011)}

Energy dissipated by the Object Tracking Sensor Net-work (OTSNs) is addressed by the author in this study. OSTN is considered as one of the most energy consuming application of WSN. The author proposed a prediction based tracking Technique using Sequential Patterns (PTSPs) which implements a novel approach in its pre-diction mechanism and design to achieve significant reductions in the energy dissipated by the OTSNs while maintaining acceptable missing rate levels. The performance metrics uses in this study are-Energy Consumption, which is reduced And Missing Rate, which is actable for the network. In this study a prediction based algorithm is use which predicts the next position of target; if the movement of target is random then it is difficult to predict the next position. There is a very limited focus on the energy lost in this study it needs to be discussed more.

\subsection{An Energy-Efficient Tracking Algorithm Based on Gene Expression Programming in Wireless Sensor Networks (Dai et al., 2009)}

In this study problem addressed is Energy consumption to tracking moving target in Sensor Network. In this study the author proposed Incremental Gene Expression Programming (GEP). That discovers the moving patterns of moving objects for turn on/off some sensor nodes at certain time to save energy. The performance metrics discussed in this study areTracking efficiency; which is improved and lifetime; which is increases about $25 \%$ as compare to other algorithm. It needs to be discussed briefly about the prediction error related to this study.

\subsection{Low-Density Wireless Sensor Networks for Localization and Tracking in Critical Environments (Cenedese et al., 2010)}

The problem of localizing and tracking mobile nodes acting in a fixed Wireless Sensor Network (WSN) is addressed in this study. This study is focused on the design of a cheap and easy-to-deploy architecture for critical infrastructures. The author proposed a strategy based on an empirical map of the Received Signal-Strength (RSS) distribution that is generated by the WSN. This approach results in being well suited for low-density setups and critical environments. The performance metrics uses in this study are-accuracy, packet drop and node failures. This study use Received Signal Strength (RSS) for Localization and multipath fading, reflections, diffraction, interference are not taking in account while they affect the RSS.

\section{PROBLEMS OF EXISTING WORKS}

Over all there is no explanation of effect on target tracking when topology is changed. There is no algorithm which decides the exact path of moving node in the net-work. Most of the papers discussed about the energy and lifetime, none of them briefly discussed about another performance metrics like throughput, delay, bandwidth utilization and PDR. There is no any work on fading, interference and blocking probability.

\section{PROPOSED SOLUTIONS}

\subsection{Solution for Deciding Exact Path}

For deciding the exact path of target we have to monitor the signals from the target. There are four localization techniques which are angle of arrival (AoA; an estimate of the relative angles between nodes), the time of arrival (ToA; the time that is taken by the radio signal to propa-gate from one node to another), the time difference of arrival (TDoA; the time interval between the reception of a radio signal and an ultrasound that is emitted by a beacon) and the RSS (an index of the received signal power) (Cenedese et al., 2010) and for tracking moving object there is a prediction based target tracking approach (Samarah et al., 2011) in which the next position of target is predicted. Now if we use these algorithms AoA, ToA, TDoA and RSS for predicting the next position then we are able to find exact path of moving target.

\subsection{Solution for Fading and Interference Between Signals}

Fading and interference are the major issues of wireless communication, so it may affect the data send by sensor nodes. We should use cluster based architecture (Khin, 2008) in which each cluster have their cluster heads. Then we arrange a priority table at base station for $\mathrm{CH}$ based on RSS of target. The $\mathrm{CH}$ having higher Priority will be able to send data to base station. For example if a target enter in a densely deployed sensor nodes then the $\mathrm{CH}$ which is nearer to it have high priority due to received signal strength which is high. At that time only single $\mathrm{CH}$ is able to send data to BS. That removes interference due to another cluster heads. Now if the selected $\mathrm{CH}$ sends data to BS then there will be problem of fading, now for overcome the problem of fading again we make an arrangement of priority table of RSS from selected 
cluster head. Here the signal have high strength will be accepted rest will be rejected. This algorithm will be able to overcome problem of fading at BS.

\subsection{Solution for Delay}

Delay of signal is another issue of Wireless sensor net-work. It is not a big issue for small area network because Base Station is located to near the sensor and node are directly contact base station if it is in the communication range of sensor node. But if base station is not in communication range of sensor then the signal might be delayed. For reduce delay first we reduce the size of signal by using compression algorithm ( $\mathrm{Li}$ and Ren, 2011). In this method the cluster head compress data which received from member nodes as a compact structure with these three entries. 1. Mean; which defined the mean value of data from all member. 2. Bitmap; which is a map indicating if the sensed data of a sensor node can be approximated to mean within a given compressing error bound ' $\varepsilon$ '. 3. Variance; which is an active array to store the variance of data when it falls more than the specified error constraint ' $\varepsilon$ ' away from mean. If size of data is small then it takes less time to reach destination (Base Station).

\section{CONCLUSION}

For final solution we can combine all three proposed solution for better performance. Each solution is able to solve only one problem if we combine all three solutions then it we will be able to overcome three problems.

In phase-1, we use cluster based architecture (Khin, 2008) and design a priority table for both sensor node and cluster head for overcoming the interference. In phase-2, we use prediction based target tracking approach (Samarah et al., 2011) for tracking target based on four localization techniques (AoA, ToA, ToDA and RSS) (Cenedese et al., 2010). One of the four localization techniques (AoA, ToA, ToDA and RSS) (Cenedese et al., 2010) can be adaptively chosen depending on the network size for generating priority table. In phase-3, we reduce the size of signal by using compression algorithm ( $\mathrm{Li}$ and Ren, 2011) for reducing delay. Finally data will send from sensor node to base station via cluster head based on priority table.

\section{ACKNOWLEDGEMENT}

We thank all the people who have helped us in bringing this study out successfully. The first author conveys sincere gratitude to the guide of this project.

\section{REFERENCES}

Cenedese, A., G. Ortolan and M. Bertinato, 2010. Lowdensity wireless sensor networks for localization and tracking in critical environments. IEEE Trans. Vehicular Technol., 59: 2951-2962. DOI: 10.1109/TVT.2010.2049277

Dai, S., C. Tang, S. Qiao, Y. Wang and H. Li, 2009. An energy-efficient tracking algorithm based on gene expression programming in wireless sensor networks. Proceedings of the International Conference on Information Science and Engineering, Dec. 26-28, IEEE Xplore Press, Nanjing, pp: 774-777. DOI: 0.1109/ICISE.2009.259

Fuemmeler, J.A. and V.V. Veeravalli, 2008. Smart sleeping policies for energy efficient tracking in sensor networks. IEEE Trans. Signal Proc., 56: 2091-2101. DOI: 10.1109/TSP.2007.912265

Li, J. and Q. Ren, 2011. Compressing information of target tracking in wireless sensor networks. Wirel. Sensor Netw., 3: 73-81.

Khin, T.S., 2008. Increasing lifetime of target tracking wireless sensor networks. World Acad. Sci. Eng. Technol., 44: 440-440.

Manisekaran, S.V. and R. Venkatesan, 2012. Distributed cluster based routing technique with multiple sinks for wireless sensor networks. Am. J. Applied Sci., 9: 1242-1250. DOI: 10.3844/ajassp.2012.1242.1250

Raza, M.T., G. Bag, S.W. Yoo and K.H. Kim, 2009. Dead reckoning based target tracking in wireless sensor networks. Electr. Telecommun. Res. Instit. South Korea Inform. Commun. DOI: 10.1145/1851340.1851342

Samarah, S., M. Al-Hajri and A. Boukerche, 2011. A predictive energy-efficient technique to support object-tracking sensor networks. IEEE Trans. Veh. Technol., 60: 656-663. DOI: 10.1109/TVT.2010.2102375

Tua, S.C., G.Y. Changa, J.P. Sheub, W. Li and K.Y. Hsieh, 2009. Scalable continuous object detection and tracking in sensor networks. J. Parallel Distributed Comput., 70: 212-224. DOI: 10.1016/j.jpdc.2009.12.001

Wang, X., S. Wang, D.W. Bi and J.J. Ma, 2007. Distributed peer-to-peer target tracking in wireless sensor networks. Sensors, 7: 1001-1027. DOI: $10.3390 / \mathrm{s} 7061001$

Watfa, M.K. and S. Commuri, 2006. An energy efficient approach to dynamic coverage in wire-less sensor networks. J. Netw. 